Pecunia Monográfico 2011, pp. 157-178

\title{
Valoración del derecho de usufructo del accionista
}

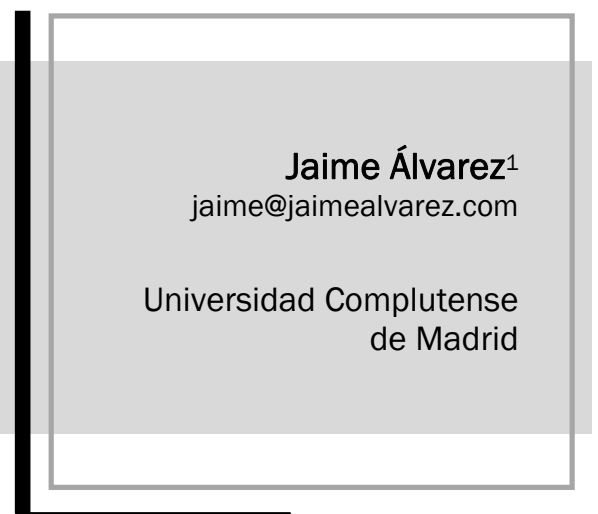

\section{Resumen}

La valoración de los títulos de propiedad de una sociedad se fundamenta, básicamente, en una doble vía complementaria, la vía patrimonial o estimación del valor de los activos que la empresa utiliza, ya sean tangibles o intangibles, o la vía de generación de rentas financieras en el futuro. ¿Qué ocurre si los titulares de los derechos patrimoniales no coinciden con los titulares de los derechos a las rentas que la empresa genere? Esto ocurre cuando el pleno dominio de los títulos de propiedad se divide en los derechos de nuda propiedad y usufructo.

Palabras clave: Valoración de empresas; Derechos reales; Usufructo.

\section{Abstract}

The shareholder value is one of the most important issues in finance as the majority of business merger and acquisitions seeks the right value for each shareholder. The total amount of value transacted in international $M \& A$ is high enough that it is much important to know exactly which the drivers of this value are.

In general, the shareholder owns both rights, what means that he owns the plain property of shares. In instance the problems arises when the usufruct right holder is not the same than the naked property right holder, this is the case in which the plain property is divided into usufruct and naked property.

This paper sets out these rights and values them.

Keywords: Equity valuation; Actuarial value; Usufruct.

${ }^{1}$ Departamento Economía Financiera y Contabilidad III, Facultad de Ciencias Económicas y Empresariales, Universidad Complutense de Madrid, Campus Somosaguas, Pozuelo de Alarcón, 28224 (Madrid). 


\section{INTRODUCCIÓN²}

La definición y análisis de los derechos reales de Usufructo y Nuda Propiedad se fundamenta en la repercusión de éstos sobre un amplio conjunto de situaciones jurídicas, valorativas y fiscales; en este sentido la diferenciación del valor correspondiente al usufructuario así como al nudo propietario es de vital importancia en cuestiones de compraventa de activos.

La aproximación al concepto de valor se caracteriza, sobre todo, por un elevado componente subjetivo $y$, de hecho, es muy común definir la valoración de activos o empresas como un arte; por ejemplo, ante dos empresas, a priori, similares se pueden obtener conclusiones diferentes. No obstante, el cálculo del valor empresarial persigue la obtención del valor de los activos, tangibles e intangibles, que componen el patrimonio de la empresa y, para ello, el analista cuenta con un completo elenco de herramientas que le permiten objetivar, en la medida de lo posible, su conclusión.

Lo que hace variar el resultado al que llega el analista es, por un lado, el objetivo de la valoración y, por otro, las circunstancias inherentes a la situación en la que se encuentra la empresa -activa o en liquidación, con o sin endeudamiento, el riesgo de negocio, etc.-. El analista debe ponderar todos los factores que pueden modificar la conclusión final y establecer las diferentes hipótesis metodológicas que conduzcan a obtener la mejor aproximación a la realidad acorde al objetivo planteado inicialmente.

Como se ha comentado anteriormente, el proceso persigue obtener el valor de los activos con los que cuenta la empresa siendo imprescindible diferenciar, inicialmente, entre el valor de la empresa en su conjunto y el valor de los títulos de propiedad representados en acciones o participaciones sociales.

Esta diferenciación permite enfocar el procedimiento de valoración ya que conceptualmente se identifica el valor de la empresa como el valor de los bienes y derechos con los que cuenta la empresa sin tener en cuenta la estructura financiera de la misma, es decir, el activo empresarial que la empresa utiliza en su función de producción. En el segundo caso, el valor de las acciones o participaciones se obtiene al tener en consideración la financiación de dichos activos puesto que el valor de los títulos de propiedad es el resultado de sustraer al valor de los activos el de las deudas u obligaciones que figuran en el pasivo exigible.

Esta clasificación es importante ya que, generalmente, el objetivo final es conseguir el valor de los títulos de propiedad por ser objeto de transmisiones o compraventas en general, derivadas de procesos de fusión, absorción, diversificación, liquidación, expropiaciones, sucesiones, donaciones, etc.

Obtener el valor tanto de la empresa en su conjunto como de los títulos de propiedad de la entidad jurídica particular está fundamentado en la metodología de valoración que se establece en torno a dos vías complementarias; por un lado la vía patrimonial y, por otro, el análisis de las rentas económicas a nivel general o de liquidez a nivel particular.

${ }^{2}$ Ver Miller y Modigliani (1961), Poterba (1986), Fama (1990), Harris (1991). 
La valoración patrimonial hunde sus raíces en el análisis del balance empresarial donde están representados los bienes y derechos así como las deudas y obligaciones que tiene la empresa que han de estar claramente identificados para su correcta valoración. En una primera aproximación llegamos al valor contable de los activos como representación del valor de la empresa. También llegamos al valor teórico de los títulos caracterizado como el valor de los fondos propios y calculado como la diferencia entre el valor contable de los activos menos el valor contable de las deudas. Esta aproximación sirve como referencia en algunos casos $\operatorname{concretos}^{3}$ y como punto de partida en el análisis de valoración.

$$
V_{F P}=V_{A}-V_{D}
$$

La segunda vía de valoración es aquella que tiene en cuenta las rentas de la empresa y que implica descubrir lo que realmente genera la empresa, a partir de las cuentas anuales de pérdidas y ganancias, y extrapolarlo hacia un futuro próximo para obtener una idea aproximada del camino que seguirá en los próximos años. A nadie se le escapa que esta previsión puede no coincidir con la realidad futura de la empresa y, evidentemente, no está exenta de incertidumbre que deriva en errores de valoración.

En cuanto al tipo de renta que se toma en consideración podemos hablar de beneficios, dividendos o flujos de caja, siendo el analista quien pondera los diferentes conceptos que componen la cuenta de resultados de cara a obtener la imagen más fidedigna de las rentas que la empresa genera mediante su actividad económica.

Un concepto clave en el análisis de la renta es la tasa de descuento que enlaza otros conceptos como el coste de capital, el riesgo económico y financiero, el plazo de la inversión, costes de oportunidad, etc. Es un elemento clave del análisis porque es la pieza que establece el valor actual de lo que la empresa va a generar en el futuro y, por tanto, su concreción en uno u otro valor puede modificar las conclusiones finales de la valoración.

En resumen, la valoración de una empresa persigue obtener el valor de los activos en general y de las participaciones sociales en particular, a través de una doble vía patrimonial y de análisis de rentas, pero iqué ocurre cuando el titular de los derechos patrimoniales no coincide con el titular de las rentas?... Esta situación se observa cuando el derecho de propiedad está dividido en los derechos reales de usufructo y nuda propiedad.

\section{LOS DERECHOS DE PROPIEDAD: USUFRUCTO Y NUDA PROPIEDAD}

La definición del derecho de propiedad ha sido un asunto controvertido durante un largo período de tiempo, hasta el punto de que no hay un concepto unívoco sobre el mismo como tampoco lo hay sobre la terminología a utilizar (Morán, 2002).

${ }^{3}$ Desde el punto de vista impositivo. 
No obstante, la propiedad, según el artículo 348 del Código Civil (CC), confiere a su titular el derecho a gozar y disponer de una cosa sin más limitaciones que las establecidas en las leyes; en cualquier caso, es evidente que el derecho de propiedad garantiza la percepción de unos frutos, así como la facultad de goce y disposición de los bienes.

La propiedad es uno de los derechos económicos más importantes en la actualidad y, como tal, viene recogido en nuestra Constitución (CE), en el artículo 33, donde se especifica que "se reconoce el derecho a la propiedad privada y a la herencia, la función social de estos derechos delimitará su contenido de acuerdo con las leyes y nadie podrá ser privado de sus bienes o derechos sino por causa justificada de utilidad pública o interés social, mediante correspondiente indemnización y de conformidad con lo dispuesto por la leyes".

Sin embargo, este derecho de propiedad puede dividirse en dos componentes de amplio uso en nuestro ordenamiento $y$, en consecuencia, nos podemos encontrar que el derecho pleno de propiedad esté desmembrado en los derechos reales de usufructo y nuda propiedad, en otras palabras, el pleno dominio está conformado por la posesión y el dominio propiamente dicho.

\subsection{Nuda propiedad}

La nuda propiedad es un derecho real que confiere a su titular la mera propiedad sobre una cosa pero no el derecho a disfrutar o a percibir los frutos de la misma, es decir, ostenta el dominio sobre la cosa pero no la posesión.

El nudo propietario tiene derecho a recuperar la cosa en buen estado a la extinción del usufructo, es decir, puede realizar toda acción encaminada a la conservación de la cosa así como las reparaciones, ordinarias o extraordinarias que hicieran falta previo requerimiento al usufructuario y, evidentemente, sin causarle perjuicio (Art. 500-503 CC). También tiene derecho a disponer de la cosa, es decir, se puede realizar una operación de compraventa de la nuda propiedad, constituir hipoteca e incluso imponer servidumbres si éstas no perjudican al usufructo.

En el caso específico de los títulos de propiedad de la empresa la legislación reserva al nudo propietario el derecho a ejercer los derechos políticos de las mismas, aunque esto puede depender de lo contenido en el título constitutivo del usufructo.

En otro sentido, el nudo propietario está obligado a entregar la cosa, no alterar la forma y sustancia de la misma ni hacer nada que perjudique al usufructuario, pagar las contribuciones sobre el capital y en el caso de no realizar las reparaciones indispensables se faculta al usufructuario a realizarlas y a exigir del propietario el aumento de valor derivado de las mismas.

Por tanto la nuda propiedad es un derecho limitado al dominio de la cosa si bien esta limitación puede ser por un reducido periodo temporal y, en cualquier caso, a la extinción del derecho real complementario. La limitación del pleno dominio se genera 
por la existencia de otro derecho real, correspondiente a un tercero, denominado usufructo.

\subsection{Usufructo}

El usufructo es un derecho real de goce o disfrute de una cosa. Implica la posesión pero no la propiedad o dominio. Puede utilizarse y disfrutarse, es decir, obtener sus frutos, tanto en especie como monetarios pero no se es el dueño de la cosa. Por ello no podrá enajenarse ni disminuirse sin el consentimiento del propietario.

En nuestro ordenamiento normativo quedan ampliamente recogidos los derechos reales de propiedad, artículos 467 y siguientes del CC, y respecto a los títulos de propiedad de empresas en los artículos 35 y siguientes de la Ley 2/1995, de 23 de marzo, de Sociedades de Responsabilidad Limitada (LSRL) y artículos 57 y siguientes del Texto Refundido de la Ley de Sociedades Anónimas (LSA), aprobado por Real Decreto Legislativo 1564/1989, de 22 de diciembre.

\subsubsection{Definición}

En el Código Civil, se regula el usufructo en cuatro secciones que comprenden los artículos 467 al 522; En la Sección Primera, Artículo 467 se define el usufructo como el derecho a disfrutar los bienes ajenos con la obligación de conservar su forma y sustancia, a no ser que el título de su constitución o la ley autoricen otra cosa. Es un derecho real sobre cosa ajena con carácter mueble o inmueble y sometido a límites temporales.

Este derecho se constituye por ley ${ }^{4}$, por prescripción ${ }^{5}$ y por acuerdo entre las partes por actos inter vivos o en última voluntad mortis causa (Art. 468).

En el caso de acuerdo entre las partes por actos inter vivos la constitución del usufructo puede ser por enajenación del usufructo y reserva de la nuda propiedad o bien atribuir a una persona el usufructo y a otra la nuda propiedad. En los acuerdos de última voluntad, el testador puede optar por legar el usufructo y dejar la nuda propiedad en herencia o, al contrario, instituir herederos en usufructo legando la nuda propiedad.

El título constitutivo del usufructo determina los derechos y obligaciones del usufructuario aunque, en su defecto, el CC lo establece en sus Secciones Segunda y Tercera, Artículos 471 y siguientes.

\subsubsection{Derechos del usufructuario (Sección Segunda, Art. 471-490)}

El usufructuario tiene derecho a percibir los frutos, ya sean de carácter natural o industrial y si éstos están pendientes al comienzo del usufructo entonces pertenecen al

\footnotetext{
${ }^{4}$ Es el caso del usufructo viudal.

${ }^{5}$ Regulación de la usucapión del derecho de usufructo a los artículos 1930 y siguientes del CC.
} 
usufructuario mientras que si lo están al término del usufructo entonces pertenecerán al propietario.

También tiene derecho a percibir los frutos civiles que se entienden percibidos día por día y pertenecen al usufructuario en proporción al tiempo que dure el usufructo.

En relación al objeto de este trabajo es importante resaltar el artículo 475 del CC según el cual si el usufructo se constituye sobre el derecho a percibir una renta periódica ya sea en metálico o intereses de obligaciones o títulos al portador, se considerará cada vencimiento como productos o frutos de aquél derecho. Así mismo tendrán la misma consideración los beneficios de una explotación industrial o mercantil cuyo reparto no tuviese vencimiento fijo.

También es interesante lo dispuesto en el artículo 480 del CC por el que el usufructuario podrá, además de utilizar la cosa por sí mismo, arrendarla o incluso enajenar su derecho. No obstante todos estos contratos celebrados por el usufructuario quedarán resueltos al fin del usufructo.

\subsubsection{Obligaciones del usufructuario (Sección Tercera, Art. 491-512 CC)}

El usufructuario está obligado a la formación de inventario y la constitución de fianza (Art. 491, 492 y 493), estando exceptuado del deber de fianza el vendedor o donante que reserve el usufructo de la cosa vendida o donada, también se exceptúa a los padres usufructuarios de los bienes cuya nuda propiedad recae en los hijos así como el cónyuge sobreviviente respecto de la cuota legal usufructuaria ${ }^{6}$. Una vez prestada la fianza tendrá derecho a todos los productos desde el día en que se constituyó el usufructo (Art. 496).

En caso de no constituirse la fianza cuando se deba, el propietario puede exigir que la cosa, si son efectos públicos, títulos de crédito nominativos o al portador, se convierta en inscripciones o se depositen en un Banco o establecimiento público y que los capitales o sumas en metálico y el precio de la enajenación de los bienes muebles se inviertan en valores seguros perteneciendo el interés generado al usufructuario. El propietario también puede retener los bienes del usufructo en calidad de administrador entregando al usufructuario el producto líquido deducida la retribución convenida o judicialmente señalada.

Las cargas y gravámenes relativos a los frutos serán de cuenta del usufructuario por la totalidad de la duración del usufructo mientras que aquellas que se impongan sobre el capital serán a cargo del propietario. Se dispone que en caso de que dichas cargas sean abonadas por aquél a quien no corresponda entonces se generará un derecho correspondiente.

${ }^{6}$ Redactado por la Ley 11/1.981, del 13 de mayo (B.O.E. del 19 de mayo), de modificación del Código Civil en materia de filiación, patria potestad y régimen económico del matrimonio. 


\subsubsection{Extinción del usufructo}

Finalmente, en la Sección Cuarta (Art. 513-522), se regula la extinción del usufructo determinando que las causas serán 1) por muerte del usufructuario, 2) por expirar el plazo o condición resolutoria determinada en el título constitutivo, 3) cuando el usufructo y la propiedad recaen en una misma persona, 4) por renuncia del usufructuario, 5) por la pérdida total del objeto de usufructo $\left.{ }^{7}, 6\right)$ por la resolución del derecho del constituyente o 7) por prescripción. El usufructo constituido a favor de un grupo de personas no se extinguirá hasta que fallezca la última persona viva.

En el supuesto de una expropiación por causa de utilidad pública, el artículo 519 del CC determina que el propietario estará obligado a la subrogación con otra cosa de igual valor y análogas condiciones o bien abonar al usufructuario el interés legal del importe de la indemnización por todo el tiempo que deba durar el usufructo.

Por último, añadir, que el usufructo no se extingue por el mal uso de la cosa usufructuada aunque si se causa un considerable perjuicio al propietario éste puede pedir la restitución de la cosa estando obligado a entregar al usufructuario lo percibido de la cosa menos su retribución como administrador.

\subsubsection{Usufructo en las Sociedades Mercantiles}

El artículo 57 de la LSA determina que la constitución de derechos reales limitados sobre las acciones procederá de acuerdo con el Derecho Común. Tratándose de acciones nominativas, la constitución de derechos reales podrá efectuarse por medio de endoso acompañado, según los casos, de la cláusula "valor en garantía" o "valor en usufructo" o de cualquier otra equivalente.

El artículo 36.1 de la SRL y el 67.1 de la LSA establecen que la cualidad de socio reside en el nudo propietario y, por tanto, ostentará los derechos inherentes al mismo salvo lo dispuesto en los estatutos $y$, en todo caso, el derecho a los dividendos acordados por la sociedad durante el usufructo, quedando el usufructuario obligado a facilitar al nudo propietario el ejercicio de estos derechos.

Respecto a la liquidación del usufructo y al derecho preferente de suscripción se remite a lo dispuesto en los artículos 68 y 70 de la LSA. Al finalizar el usufructo se podrá exigir al nudo propietario el incremento de valor experimentado por las acciones usufructuadas que corresponda a los beneficios propios de la explotación de la sociedad integrados durante el usufructo en las reservas expresas que figuren en el balance de la sociedad cualquiera que sea la naturaleza o denominación de las mismas.

Disuelta la sociedad durante el usufructo, el usufructuario podrá exigir del nudo propietario una parte de la cuota de liquidación equivalente al incremento de valor de las acciones usufructuadas previsto en el apartado anterior. El usufructo se extenderá al resto de la cuota de liquidación.

\footnotetext{
${ }^{7}$ Si la pérdida no es total, el derecho de usufructo continua en la parte restante (Art. 514).
} 
Si las partes no llegaran a un acuerdo sobre el importe a abonar en los supuestos previstos en los dos apartados anteriores, este será fijado a petición de cualquiera de ellas y a costa de ambas, por los auditores de la sociedad y, si ésta no estuviera obligada a verificación contable, por el auditor de cuentas designado por el Registrador mercantil del domicilio de la sociedad.

Una cuestión importante es la relativa a los derechos de suscripción preferente ya que, según el artículo 70.1, si el nudo propietario no ejercita o enajena el derecho de suscripción sobre el aumento de capital de la sociedad antes de diez días a la extinción del correspondiente plazo entonces el usufructuario está legitimado para vender esos derechos o bien suscribir las correspondientes acciones; en el punto 2 se establece que el usufructo comprende el importe derivado de la enajenación de los derechos de suscripción; en el punto 3 se dispone que el usufructo se extiende a las acciones conseguidas mediante los derechos de suscripción mientras que no comprende el resto de acciones suscritas cuya plena propiedad corresponde a quien desembolse su importe; el punto 4 establece lo mismo para los casos de emisión de obligaciones convertibles en acciones y, por último, el punto 5 extiende el usufructo a la emisión de nuevas acciones con cargo a beneficios o reservas aunque corresponden al nudo propietario.

\section{VALORACIÓN}

En la evaluación empresarial se atribuye valor a un conjunto de factores productivos y financieros que se coordinan bajo una misma organización, generando unos determinados efectos sinérgicos (Demsetz, 1967). Estos elementos conforman el balance empresarial y ante una determinada composición permiten generar bienes o servicios que, con el tiempo, revierten económicamente en la entidad.

El valor es un concepto económico que se fundamenta en lo que un agente estaría dispuesto a desembolsar por un determinado producto, servicio o activo en un mercado eficiente, es decir, en un mercado donde el precio se forma teniendo en consideración toda la información disponible sobre el bien que se intercambia.

Una definición de mercado eficiente es la que establece que la competencia entre los agentes que participan en el mercado deriva en una situación de equilibrio en la que el precio es una buena estimación del valor intrínseco (Suárez, 2003). En otras palabras, un mercado ineficiente es aquel en el que el precio no se corresponde con el valor de los bienes intercambiados.

Es importante resaltar que la motivación de los agentes que participan en una transacción es el aprovechamiento de valor, o falta de valor, oculto a la contraparte. Existirá una posición vendedora cuando la opinión sobre el valor del objeto de la transacción sea menor que el precio ofrecido y habrá un comprador cuando su opinión sobre el valor del objeto de la transacción sea superior al precio de la misma. A nivel empresarial estas transacciones se pueden especificar con el intercambio de activos productivos o reales, instrumentos financieros, organizaciones en su conjunto, etc. 
En definitiva, el objetivo perseguido por la valoración empresarial es ofrecer información detallada sobre la posibilidad de la empresa para seguir cumpliendo los fines de la misma o bien para establecer el valor de las partes individualmente consideradas. Las partes pueden tener razones fundamentadas para realizar la transacción y todo ello dependerá, en gran medida, de la información que mantienen en su poder.

La valoración es un proceso subjetivo sobre la cantidad económica que debería desembolsarse para la adquisición de un determinado bien económico en función de la información relativa al mismo. En ausencia de información la percepción del agente puede ser incorrecta y por tanto no se estimará correctamente el valor de aquello que se quiera intercambiar. La competencia es un factor clave ya que genera el equilibrio necesario en el mercado al configurar los precios entre todos los participantes que manejan la información.

En el presente trabajo se intenta ampliar la información disponible para la correcta valoración de los títulos de propiedad cuando concurren las circunstancias de separación entre los titulares de los derechos de propiedad o nuda propiedad y los titulares de los derechos a las rentas o usufructo.

\subsection{El valor de los activos, la empresa y los títulos de propiedad}

Sin intención de realizar una amplia exposición del concepto de valor, éste está relacionado con las rentas que un activo pueda generar en un futuro. La teoría de valoración y selección de inversiones se fundamenta en el bien conocido criterio del Valor Capital o Valor Actual Neto de una inversión, donde los flujos de renta, o más concretamente, los flujos de caja asociados a una inversión se actualizan a una determinada tasa de descuento lo cual nos conduce hacia la aceptación o rechazo de la inversión al comparar el valor actual de dichos flujos de caja con el desembolso necesario para realizarla.

$$
V A N=-A+\frac{C_{1}}{\left(1+k_{1}\right)}+\frac{C_{2}}{\left(1+k_{1}\right)\left(1+k_{2}\right)}+\ldots .+\frac{C_{n}}{\left(1+k_{1}\right)\left(1+k_{2}\right) . .\left(1+k_{n}\right)}=-A+\sum_{j=1}^{n} C_{j} \prod_{j=1}^{n}\left(1+k_{j}\right)^{-1}
$$

Son, por tanto, las rentas futuras que el activo es capaz de generar, el pilar fundamental de la valoración de activos, siempre teniendo en cuenta la tasa de descuento oportuna que en este caso se asimila al coste del capital propio de cada organización o inversor. Si éste último se considera constante en los sucesivos años $k_{i}=k_{j} \quad \forall i \neq j$, entonces

$$
V A N=-A+\frac{C_{1}}{(1+k)}+\frac{C_{2}}{(1+k)^{2}}+\ldots .+\frac{C_{n}}{(1+k)^{n}}=-A+\sum_{j=1}^{n} \frac{C_{j}}{(1+k)^{j}}
$$

La valoración de activos implica la consideración subjetiva de la generación de rentas para un posible inversor en el sentido de que cada inversor podrá utilizar el activo correspondiente para obtener unas rentas adaptadas a su estructura de producción. Como cada agente inversor tiene una determinada función de producción, las rentas 
que el activo vaya a generar en el futuro dependerán de los factores determinantes de dicha función de producción, con lo que se llega a una situación en la que cada agente tendrá una propia, y diferente, estimación de los flujos de caja asociados a la utilización de cualquier activo.

Es, precisamente, está estimación subjetiva de las futuras rentas que se van a obtener actualizadas a la correspondiente, y también específica, tasa de descuento, la explicación de porque unos agentes podrán estar interesados en la adquisición de un activo mientras que otros no lo estarán.

La comparación entre el valor actual de las rentas, que cada agente estima que va a conseguir con la adquisición de un determinado activo, y el desembolso necesario para adquirirlo determina el valor actual neto de la inversión; cuando dicho valor es positivo la inversión interesa realizarla puesto que se está creando valor.

La empresa no es otra cosa que un conjunto de activos productivos, tangibles o intangibles, que agregadamente configuran un activo aumentado, con unas rentas futuras estimables y, en consecuencia, valorable. El valor de la empresa se reduce al valor de los activos que la componen en tanto en cuanto son estos mismos activos los que generan las rentas que determinan la cuenta de resultados de la empresa.

El valor de los activos que utiliza una empresa se puede aproximar mediante la información contable presente en las cuentas anuales, es decir, el valor de los activos empresariales reflejado en el balance contable. Sin embargo, como se ha precisado, este valor es una aproximación que generalmente establece una base para el proceso de valoración de los activos empresariales. La razón de ello estriba en la carencia de información sobre los valores de mercado que ofrece la contabilidad actual. El criterio de imputación del valor de adquisición así como la disminución de dicho valor por el efecto de la amortización, o incluso la utilización de diferentes métodos de valoración de circulante conlleva una situación en la que, en algunos activos determinados, el valor de mercado no quede recogido en el valor contable. Por tanto, en muchas ocasiones el valor contable no es reflejo del valor de mercado de los activos.

Aunque tampoco el valor de mercado de los activos que configuran el sistema productivo empresarial es, necesariamente, el valor de los mismos para la empresa. La explicación evidente de esta afirmación descansa en la previa definición de valor actual neto de una inversión; mientras que el valor de mercado de un activo vendrá dado por su valor de adquisición, es decir el desembolso necesario para su adquisición, el valor para la empresa es mayor dado que el criterio de selección de la inversión a estimado un valor actual de la inversión superior a su desembolso.

Por tanto, el valor de los activos para una empresa es superior al valor de mercado de los mismos puesto que en caso contrario serían activos que no deberían incorporarse al sistema productivo al presentar un valor inferior al desembolso necesario para su adquisición. 
Este valor podríamos obtenerlo individualmente analizando la generación de rentas financieras asociadas a cada activo o bien, a nivel agregado, analizando las rentas financieras en su conjunto descontadas al coste promedio de todas las fuentes de financiación de la empresa (Modigliani y Merton, 1958). Este coste promedio es la tasa de rentabilidad exigida por todos los titulares de las fuentes de financiación en función de su ponderación en la financiación total de la empresa.

Para ello, hay que obtener la cuantía de las rentas financieras agregadas así como su estimación futura para posteriormente descontarlas al coste promedio de las fuentes de financiación. En la valoración de los flujos monetarios o rentas el instrumento fundamental es la cuenta de pérdidas y ganancias, complementada -al igual que en el caso anterior- por la memoria.

Del análisis de la cuenta de resultados se desprende la capacidad que tiene la empresa objeto de estudio para la generación de valor, tanto en el presente como en el futuro, dado que es un elemento en continua evolución. La determinación de los estados previsionales otorga al analista una herramienta de anticipación a la realidad empresarial y por tanto un componente de valoración de elevada utilidad.

La estimación de las rentas que genera la empresa generalmente está identificada con el beneficio empresarial o con el dividendo repartido ${ }^{8}$, sin embargo, ambas medidas adolecen de cierta arbitrariedad contable al estar condicionadas a los sistemas contables internacionales y por tanto no reflejan con exactitud la realidad generadora de rentas de liquidez por parte de la empresa ${ }^{9}$. En este sentido surge el concepto de Flujo de Caja (FC) como medida más representativa de la generación de rentas futuras.

"El valor de una empresa depende únicamente de los flujos de liquidez que prometa generar en un futuro ... Por tanto, el valor descansa en los flujos de caja que la empresa es capaz de generar". ${ }^{10}$

"El valor de las acciones de una empresa proviene de la capacidad de la misma para ganar dinero (flujos) para los propietarios de las acciones". ${ }^{11}$

Es necesario aclarar que esta metodología calcula el valor de la empresa a través del descuento de flujos por lo que hay que abordar dicho análisis teniendo en cuenta la tasa de descuento apropiada en función del concepto que se utilice. En concreto el método de valoración más utilizado es el Descuento de Flujos de Caja (DFC) que comprende las

${ }^{8}$ El modelo de Gordon-Shapiro establece su base matemática en los dividendos con y sin crecimiento, ver Gordon y Shapiro (1959) y Gordon (1956).

${ }^{9}$ En el caso de valoraciones con un horizonte temporal finito el método de Descuento de Beneficios se presenta como el más apropiado, Sougiannis y Penman (1996), aunque "puede ser un error considerar dicha aproximación” y "son resultado de la aplicación de determinadas normas de contabilidad que pueden ser adoptadas arbitrariamente por la dirección de la empresa y por tanto enfocadas a cumplir con criterios estrictamente contables” en Fernández (2000 y 2003).

10 En Mascareñas (2005).

11 En Fernández (2000). 
siguientes fases: (1) estimar los FC futuros, (2) descontar las estimaciones de FC al valor actual al coste apropiado como el Coste del Capital Medio Ponderado y (3) ajustar por grado de control.

Sin embargo, para conseguir una primera aproximación se utilizan métodos patrimoniales como el valor neto contable, valor neto contable corregido, valor sustancial o valor de liquidación (Fernández, 2000). Con base en las rentas a generar encontramos la valoración por múltiplos destacando el Ratio Precio Ganancia (Price Earnings Ratio, PER), Crecimiento Precio Ganancia (Price Earnings Growth, PEG) ${ }^{12}$, el ratio Precio Flujo de Caja (Price Cash Flow, PCF), el Precio Valor Contable (PVC), y los modelos de descuento de dividendos cuyo máximo exponente es el modelo de Gordon Saphiro.

Como el valor de cada uno de los activos de la empresa viene dado por el descuento de los flujos de caja que van a generar en el tiempo, siendo estos flujos de caja la fuente, en última instancia, del beneficio y los dividendos; este concepto implica que el valor patrimonial de la empresa es la suma del valor actual de los flujos de caja de cada inversión económica.

La diversidad en la estimación de las rentas que generará la empresa favorece múltiples posiciones compradoras y vendedoras o lo que es lo mismo genera un sinfín de posibilidades en la obtención de un valor concreto; en otras palabras, cada comprador o vendedor tendrá su propia estimación subjetiva y, por ende, su propia predisposición a la materialización del precio en las operaciones de transmisión empresarial. Se hace evidente que la diversidad en la estimación de las rentas se fundamenta en diferentes perspectivas sobre la generación de recursos por parte de la empresa lo cual viene generado por una falta o exceso de análisis en la determinación del valor actual de las rentas futuras (Brealey y Myers, 2003).

Las rentas financieras que habrán de utilizarse en estas estimaciones, y que parece existe un consenso entre los analistas, se denominan flujos de caja libres de la empresa (FCL) mientras que el coste de las fuentes de financiación se denomina coste del capital medio ponderado (CCMP).

El valor de la empresa, o lo que es lo mismo, el valor de los activos vendrá dado por la siguiente expresión, considerando el CCMP constante en el tiempo, una duración determinada en el tiempo y un valor residual de dichos activos a partir del límite temporal de proyección de los flujos de caja:

$$
V A c=\frac{F C L_{1}}{(1+k)}+\frac{F C L_{2}}{(1+k)^{2}}+\ldots .+\frac{F C L_{n}}{(1+k)^{n}}+\frac{F C L_{n+1}}{(1+k)^{n+1}}+\ldots .=\sum_{j=1}^{n} \frac{F C L_{j}}{(1+k)^{j}}+V R A C
$$

O bien, en el supuesto de variabilidad del coste del capital de la empresa, como:

12 Un relación detallada de algunos ratios utilizados en los procesos de valoración se puede encontrar en Mascareñas (2005). 


$$
V A c=\sum_{j=1}^{n} F C L_{j} \prod_{j=1}^{n}\left(1+k_{j}\right)^{-1}+V R A c
$$

Así, una vez valorados correctamente los activos obtenemos el valor de la empresa. Esta valoración nos permite establecer, posteriormente, el valor de los títulos de propiedad, que en la mayoría de las ocasiones será el objetivo final del proceso de valoración.

Ahora bien, habrá que tener en cuenta que la totalidad de activos que utiliza la empresa en su función de producción pueden ser de dos tipos, por un lado aquellos que estarán reflejados en la contabilidad y que se denominan activos tangibles y otra tipología de activos que no quedan recogidos por la contabilidad y que se denominan activos intangibles ${ }^{13}$. La estimación de valor de éstos últimos es una cuestión prácticamente de fe. Sin embargo la expresión anterior habrá de quedar modificada por la siguiente:

$$
V_{A}=V_{A T}+V_{A I} \quad[6]
$$

donde $V_{A T}$ es el valor de los activos tangibles presentes en el balance contable y $V_{A I}$ es el valor de los activos intangibles.

En cualquier caso, el valor de la totalidad de los activo podrá apreciarse mediante el análisis de las rentas que genera la empresa pues en ellas queda recogida toda la información financiera relativa a los activos que se están utilizando en la actividad empresarial.

Como ya se ha comentado, el valor de los activos, además de los pasivos, viene aproximado por el balance contable y, por tanto, es factible obtener el valor de los títulos de propiedad mediante el correspondiente a los fondos propios contables que figuran en el balance. Es decir, el valor contable de los activos menos el de las deudas nos ofrece una primera aproximación al valor de los fondos propios como estimación del valor de los títulos de propiedad.

No obstante, nos hemos referido al valor contable como una estimación sesgada del verdadero valor de los activos empresariales, lo que no ocurre, sin embargo y generalmente, con los pasivos exigibles de la empresa ya que, en la mayoría de los casos, el valor de los pasivos exigibles reflejado en la contabilidad suele ser una aproximación significativa de su valor real aunque, evidentemente, esto dependerá de las características individuales de cada uno de los títulos de deuda.

Según esta aproximación el valor de los títulos de propiedad vendrá dado por la expresión que representa la sustracción del valor de las deudas al valor de los activos, donde $V_{A}$ es el valor de los activos totales es decir, tangibles e intangibles.

13 No hay que confundir activos intangibles con inmateriales pues estos últimos sí tienen cabida en el marco contable y, por tanto, están representados en el activo del balance. Una cuestión aparte es su valoración. 


$$
V_{F P}=V_{A}-V_{D}=V_{A T}+V_{A I}-V_{D}
$$

Esta expresión es válida, en cualquier caso, tanto si los valores correspondientes lo son a nivel contable, de mercado o reales.

Por otro lado, el descuento de las rentas asociadas a dichos títulos viene dado por la actualización de los flujos de caja libres para el accionista (FCL Acc) a la correspondiente tasa de descuento que en este caso viene dada por el coste de los recursos propios o, lo que es lo mismo, la rentabilidad exigida por los propietarios acorde al correspondiente nivel de riesgo que además consideramos constante en el tiempo.

$$
V A c=\frac{F C L A c c_{1}}{\left(1+k_{e}\right)}+\ldots .+\frac{F C L A C c_{n}}{\left(1+k_{e}\right)^{n}}+\frac{F C L A c c_{n+1}}{\left(1+k_{e}\right)^{n+1}}+\ldots .=\sum_{j=1}^{n} \frac{F^{\prime} C L A c c_{j}}{\left(1+k_{e}\right)^{j}}+V R A C C
$$

Una cuestión diferente es el cálculo del valor de las participaciones sociales, ya que la empresa realiza su actividad con base en un conjunto de activos que sinérgicamente generan el resultado empresarial, desde un punto de vista económico y financiero, que dependen de una determinada estructura financiera que condiciona dicho resultado así como el valor de los fondos propios.

Dado que la valoración de los derechos de propiedad se puede realizar mediante una metodología contable patrimonial o financiera surge el inconveniente de representar con corrección dicho valor cuando el derecho de pleno dominio está desmembrado en los derechos de usufructo y nuda propiedad.

El derecho de usufructo se identifica con el derecho a recibir las rentas de la empresa mientras que el derecho de nuda propiedad se identifica con la titularidad de los activos de la misma.

\subsection{Descomposición del pleno dominio en usufructo y nuda propiedad. Fórmulas de Achard y Makeham}

La demostración matemática de la descomposición del valor del pleno dominio en los derechos de usufructo y nuda propiedad está asociada, en la matemática de las operaciones financieras, al análisis del valor financiero de los préstamos. Este análisis tiene su fundamento en las operaciones de novación y subrogación hipotecaria.

El valor de pleno dominio se identifica con el valor actual financiero de los términos amortizativos correspondiente a un préstamo cuando se establece dicho valor en función de una ley financiera externa establecida por las cambiantes condiciones del mercado, en otras palabras, es la valoración de los futuros términos amortizativos descontados a la vigente tasa de interés en el mercado para las operaciones similares (Gil Luezas y Gil Peláez, 1992).

- Así, el valor financiero del préstamo vendrá dado por la siguiente expresión: 


$$
V_{s}=\sum_{r=s+1}^{n} a_{r} \prod_{h=s+1}^{r}\left(1+i_{h}^{\prime}\right)^{-1}
$$

siendo $a_{r}$ el valor de los términos amortizativos del préstamo e $i_{h}{ }_{h}$ el nuevo tanto de interés correspondiente a las nuevas condiciones vigentes en el mercado.

Dado que el término amortizativo se puede descomponer en la cuota de amortización y la cuota de interés podemos, entonces, valorar individualmente el usufructo y la nuda propiedad. El usufructo es una figura que se identifica con el valor actual financiero de la cuota de interés mientras que el valor de la nuda propiedad lo será en base a la cuota de amortización de un préstamo cuando se establecen dichos valores en función de una ley financiera externa, en otras palabras, es la valoración individual de las futuras cuotas de amortización e interés que componen los términos amortizativos.

Así, el valor financiero del usufructo Us, vendrá dado por la expresión:

$$
U_{s}=\sum_{r=s+1}^{n} C_{r-1} i_{r} \prod_{h=s+1}^{r}\left(1+i_{h}{ }^{-1}\right.
$$

siendo $C_{r-1} i_{r}$ el valor de las cuotas de interés del préstamo mientras que el valor financiero de la nuda propiedad será el resultado de la siguiente expresión matemática:

$$
N_{s}=\sum_{r=s+1}^{n} A_{r} \prod_{h=s+1}^{r}\left(1+i_{h}^{\prime}\right)^{-1}
$$

donde $A_{r}$ es el valor de las sucesivas y futuras cuotas de amortización del préstamo.

La relación existente entre el valor del usufructo y de la nuda propiedad queda recogida en la conocida fórmula de Achard ${ }^{14}$, según la cual el valor del usufructo es equivalente al valor del cociente entre el tanto de interés anterior y el actual por la diferencia entre la reserva matemática del préstamo y el valor de la nuda propiedad, es decir,

$$
U_{s}=\frac{i}{i^{\prime}}\left[C_{s}-N_{s}\right]
$$

Por tanto, en el caso de los préstamos, el valor del pleno dominio es claramente identificable con la suma de los valores del usufructo y de la nuda propiedad.

$$
V_{s}=U_{s}+N_{s}
$$

14 Achard (1912). 
esta relación también se puede expresar, en el análisis de los préstamos, a través de la denominada fórmula de Makeham ${ }^{15}$ que es el resultado de la resolución del sistema formado por las ecuaciones [12] y [13]:

$$
V_{s}=\frac{i}{i^{\prime}}\left[C_{s}-N_{s}\right]+N_{s}
$$

\subsection{El valor de la nuda propiedad, del pleno dominio y del usufructo}

En la estimación de los valores individualizados de los componentes del pleno dominio nos vamos a centrar en la expresión [5] anteriormente descrita obteniendo el valor de la nuda propiedad como el complemento al valor del usufructo ${ }^{16}$, por tanto es necesario obtener inicialmente el valor del pleno dominio para, posteriormente, sustraer el valor del usufructo.

\subsubsection{El valor del pleno dominio}

En el epígrafe 3.2. se ha estimado el valor del pleno dominio de un préstamo como el valor financiero de los términos amortizativos del mismo teniendo en cuenta el nuevo tanto de interés de mercado para operaciones similares. Esta valoración es factible desde un punto de vista técnico siempre y cuando se tengan los valores de los parámetros de la ecuación [4].

Ahora bien, en el caso de los préstamos obtener el valor del usufructo y de la nuda propiedad es igualmente factible al estar claramente diferenciados los términos amortizativos y de interés para su descuento mediante la ley financiera externa según las ecuaciones [5] y [6], con lo que todos los valores están perfectamente identificados.

Sin embargo, en el caso de los títulos de propiedad de la empresa esta diferencia no es tan evidente ni observable como en el caso de los préstamos. En consecuencia, resulta de interés definir cuáles serán los valores asociados al pleno dominio, usufructo y nuda propiedad de los títulos de propiedad empresarial y, por tanto, los flujos de caja asociados a dichos elementos.

La valoración empresarial del pleno dominio se corresponde con lo descrito en el epígrafe 3.1 estableciéndose el correspondiente valor de los títulos de propiedad con determinada objetividad. Una vez conseguido dicho valor del pleno dominio habrá que centrarse en el cálculo de los valores complementarios de nuda propiedad y usufructo.

15 Makeham (1873).

16 El artículo 26 del Impuesto sobre Sucesiones y Donaciones establece que el valor de la nuda propiedad será equivalente a la diferencia entre el valor del usufructo y el valor total de los bienes. 


\subsubsection{Valor de la nuda propiedad}

El valor de la nuda propiedad como se define como la diferencia entre el valor de pleno dominio y el de usufructo, en este sentido se sigue la ecuación [13] anterior:

$$
N_{s}=V_{s}-U_{s}
$$

Si el valor de la nuda propiedad depende del valor del pleno dominio y del usufructo es, por tanto, necesario establecer dichos valores.

\subsubsection{El valor del usufructo}

Finalmente, debemos calcular el valor del usufructo para determinar el valor de los tres elementos que estamos analizando y para ello hay que diferenciar entre dos posibilidades; el usufructo temporal y vitalicio. En el primer caso el usufructo puede tener carácter temporal, es decir, el título constitutivo del usufructo establece una fecha límite de duración a partir de la cual revertirá al nudo propietario mientras que en el segundo caso este derecho se mantiene hasta la defunción del titular. Esta diferenciación es importante de cara a establecer los fundamentos técnicos necesarios para su correcta valoración.

\subsubsection{Usufructo temporal}

En la valoración de las acciones o participaciones sociales el cálculo del valor del usufructo debe obtenerse mediante el descuento de las rentas asociadas al mismo por el periodo de vigencia del usufructo. En términos generales, la renta económica se asimila a los dividendos estimados para los períodos futuros tal y como se establece en los artículos 36.1 de la SRL y 67.1 de la LSA.

Sin embargo, la valoración del usufructo temporal según el artículo 26 de la Ley 29/1987, de 18 de Diciembre, del Impuesto sobre Sucesiones y Donaciones se establece a razón de un $2 \%$ por cada periodo de un año con un máximo del $70 \%$, sobre el valor total de los bienes objeto de usufructo, lo cual implica que, en primer lugar hay que estimar el valor total de los bienes para posteriormente obtener el valor del usufructo. Además el límite temporal del mismo viene dado por el valor máximo porcentual que se corresponde con un periodo temporal de 35 años.

Resulta evidente que la norma fiscal que se desprende del artículo 26 adolece de la necesaria exactitud técnica si bien se caracteriza por una gran simplicidad y eficiencia al no estar muy alejada de la posible valoración realizada teniendo en cuenta la disminución de valor por cada año transcurrido hasta la edad determinada como final. Esta característica se analiza en el siguiente epígrafe.

En consonancia con el principio financiero de la actualización, si denotamos por Dj el dividendo de cada periodo futuro hasta la extinción del usufructo temporal y por $\mathrm{N}$ el tiempo de finalización de este derecho durante el que se van a percibir los 
correspondientes dividendos, entonces el Valor Actual, utilizando la correspondiente tasa de descuento Ke, viene dado por la siguiente expresión:

$$
V A=\sum_{j=0}^{N} D_{j}\left(1+k_{e}\right)^{-j}
$$

La expresión [15] se refiere al periodo desde el momento inicial $(j=0)$, dado que el artículo 496 del CC establece que el usufructuario tendrá derecho a todos los productos generados por el bien usufructuado desde el día en que, conforme al título constitutivo, se debe comenzar a percibirlos. Además dicha expresión es válida en el caso de que sea aplicable lo dispuesto en el artículo 516 del CC según el cual el usufructo concedido por el tiempo que tarde un tercero en llegar a cierta edad, subsistirá el número de años prefijado, aunque el tercero muera antes, salvo si dicho usufructo hubiere sido expresamente concedido sólo en atención a la existencia de dicha persona.

En realidad, al tratarse de un derecho real, transmisible en el caso de defunción del titular si no es de aplicación lo dispuesto en el anterior artículo, habrá que incluir en el cálculo correspondiente las probabilidades de defunción, o sus complementarias de supervivencia, para estimar el valor probable del usufructo. Así, si denotamos por $\left({ }_{t} p_{x}\right)$ las probabilidades de supervivencia de un individuo de una determinada edad (x) a favor de quien esté constituido el correspondiente usufructo y por $\left({ }_{t} q_{x}\right)$ las probabilidades de defunción podemos describir la dimensión financiera de esta corriente de flujos monetarios asociados a sus respectivas probabilidades (Gil, Heras y Vilar, 2000), así:

\begin{tabular}{|c|c|c|c|c|c|}
\hline & $\begin{array}{ll}D_{1} & 1 p_{x} \\
0 & 1 q_{x}\end{array}$ & $\begin{array}{ll}D_{2} & 2 p_{x} \\
0 & 2 q_{x} \\
\end{array}$ & ........ & $\begin{array}{ll}D_{n-1} & n-1 p_{x} \\
0 & n-1 q_{x}\end{array}$ & $\begin{array}{ll}D_{n} & n p_{x} \\
0 & n q_{x} \\
\end{array}$ \\
\hline 0 & 1 & 2 & ........ & $\mathrm{n}-1$ & $\mathrm{~N}$ \\
\hline
\end{tabular}

El valor probable de cada flujo anual se corresponde con la esperanza matemática según la siguiente expresión:

$$
E[F]=D_{j j} p_{x}+0_{j} q_{x}=D_{j j} p_{x}
$$

y, por tanto, la expresión [15] se transforma en el Valor Actual Actuarial y queda expresada como:

$$
V A A=\sum_{j=0}^{N} D_{j}\left(1+k_{e}\right)^{-j}{ }_{j} p_{x}
$$

que también se puede expresar como:

$$
V A A=\sum_{j=0}^{N} D_{j} v_{j}^{j} p_{x}=\sum_{j=0}^{N} D_{j j} E_{x}
$$


Esta segunda expresión [18] nos permite obtener el valor actual del usufructo desde una perspectiva actuarial, es decir, en función de las probabilidades de supervivencia del titular de los derechos de usufructo, a través del denominado Factor de Actualización Actuarial $\left({ }_{j} E_{x}\right)$.

Esta caracterización del usufructo es de una relevancia indiscutible al estar asociado cada flujo monetario, al que se tiene derecho, a una determinada probabilidad generando un valor medio en función de dichas probabilidades. Este valor medio nos proporciona una estimación del valor más probable asociado a una corriente de flujos aleatorios siendo una medida estadística eficiente.

En ocasiones, el usufructo se enajena a favor de un grupo de individuos y también habrá de tenerse en cuenta dicha particularidad. En este sentido el artículo 521 del CC dispone que cuando el usufructo esté constituido en provecho de varias personas vivas al tiempo de su constitución, no se extinguirá hasta la muerte de la última que sobreviviere, si bien, en el título constitutivo se puede disponer aquello que estimen conveniente las partes.

En este caso, las ecuaciones que describen el Valor Actual Actuarial del usufructo deben ser modificadas en función de que este derecho se extinga al último fallecimiento, tal y como dispone el artículo 521 o bien se extinga al primer fallecimiento si así está dispuesto en el título constitutivo.

En el primer caso, suponiendo un grupo de dos individuos con edades $x$ e $y$, la ecuación que establece el valor del usufructo es la siguiente:

$$
V A A=\sum_{j=0}^{N} D_{j}\left(1+k_{e}\right)^{-j}{ }_{j} p_{\overline{x y}}
$$

donde ${ }_{t} p_{\overline{x y}}={ }_{t} p_{x}+{ }_{t} p_{y}-{ }_{t} p_{x y}$ y ${ }_{t} p_{x y}={ }_{t} p_{x t} p_{y}$

Mientras que en el segundo caso, igualmente suponiendo un grupo de dos individuos con edades $x$ e $y$, la ecuación que describe dicho valor es:

$$
V A A=\sum_{j=0}^{N} D_{j}\left(1+k_{e}\right)^{-j}{ }_{j} p_{x y}
$$

\subsubsection{Usufructo vitalicio}

En muchas ocasiones el derecho de usufructo adquiere un carácter vitalicio, es decir un derecho que se mantiene hasta la defunción del titular, este es el caso en supuestos hereditarios donde la masa patrimonial se divide entre los derechos de nuda propiedad para los herederos directos mientras que el derecho de usufructo recae en el cónyuge superviviente. Por tanto, la valoración del usufructo vitalicio hunde sus raíces en un 
amplio elenco de posibilidades dadas por la necesidad de valorar los derechos de los diferentes titulares de la masa patrimonial y, en consecuencia, la valoración se convierte en una tarea más complicada dado que deviene en un periodo incierto de vigencia del derecho a los dividendos.

De nuevo, la valoración del usufructo vitalicio según el artículo 26.a. de la Ley del Impuesto sobre Sucesiones y Donaciones se establece que dicho valor será del $70 \%$ sobre el valor total de los bienes en el supuesto de que el usufructuario sea menor de 20 años, disminuyendo dicho porcentaje a razón de un 1\% por cada año con un mínimo del $10 \%$ sobre el valor total. Este mínimo se corresponde con la edad de 79 años a partir de la cual el usufructo toma un valor correspondiente al 10\% del valor del bien usufructuado.

Un segundo mecanismo sería obtener el valor de dichas participaciones en función del valor actual actuarial, es decir, teniendo en cuenta las probabilidades actuariales de supervivencia. Así el valor del usufructo vitalicio se obtiene mediante el descuento de los dividendos (Dj) por un periodo comprendido entre la edad del individuo y su edad de muerte.

$$
V A A=\sum_{j=0}^{W-X-1} D_{j}\left(1+k_{e}\right)^{-j}{ }_{j} p_{X}
$$

La edad de muerte o defunción es una variable estocástica que depende de una serie de factores pero que puede obtenerse de las tablas actuariales de mortalidad, así como las probabilidades de supervivencia del titular del derecho. La edad límite en las tablas actuariales es de 115 años en el caso español.

\section{CONCLUSIONES}

En la estimación del valor de los derechos reales que comprenden el pleno dominio se ha de operar desde una vía actuarial dadas las características propias del usufructo, ya que al ser un derecho inherente a su titular cuando este deja de existir aquél también lo hará.

En la estimación de los flujos financieros que habrán de tenerse en cuenta para la obtención del valor del usufructo habrá que atender a lo dispuesto en el título constitutivo del mismo. En su defecto estas rentas se asocian al dividendo entregado por la empresa con las implicaciones que de ello se derivan (una empresa que no reparta dividendo tendrá un valor nulo del usufructo).

El valor actual de la corriente de flujos asociada al usufructo está determinado por las probabilidades de supervivencia del titular del derecho y no se puede obviar esta condición en la valoración de este derecho. Así mismo, el valor complementario al usufructo, es decir, el correspondiente a la nuda propiedad vendrá determinado por los valores del pleno dominio y del usufructo. 
La inclusión de las probabilidades de supervivencia se puede conseguir mediante las tablas actuariales de defunción que son ampliamente utilizadas en el sector asegurador y que bajo la revisión realizada a finales de la década de 1990, que dio como resultado la generación de las tablas PERM/F 2000, representa con mayor exactitud la evolución actual de la población española.

\section{BIBLIOGRAFÍA}

Achard, M. (1912). "Note sur la deuxième propriété de la formule de Makeham", Institut Actuariel Frances, 22, pp 197-204.

Brealey, R. y Myers, T. (2003). Finanzas corporativas. Madrid: McGraw Hill.

Caballer, V. (1998). Métodos de valoración de empresas. Madrid: Pirámide.

Demsetz, H. (1967). "Towards a theory of property rights", The American Economic Review, 57 (2), pp. 347-359.

Fama, F. (1990). "Stock returns, expected returns, and real activity", Journal of Finance, 45, pp. 1089-1109.

Fernández, P. (2003). "Cash flow is a fact. Net income is just an opinion", IESE, Octubre 2003.

Fernández, P. (2004). "Valuing companies by cash flow discounting: Ten methods and nine theories". EFMA 2002, London Meetings.

Fernández, P. (2006). Valoración de empresas. Barcelona: Gestión 2000.

Gil Luezas et al (1991). Matemática de las operaciones financieras. Ed. UNED, Volumen II.

Gil, Heras y Vilar (2000). Matemática de los seguros de vida. Ed. Mapfre.

Gordon, M.J. y Saphiro, E. (1956), “Capital Equipment Analysis: The required rate of profit”, Mangament Science, 3 (1), pp. 102-110.

Gordon, M.J. (1959). “Dividends, earnings and stock prices”, Review of Economics and Statistics, 41 (2), 99-105.

Harris, et al (1991). "The theory of capital structure”, Journal of Finance, 46, pp. 297355.

Lamothe, P. (2003). Valoración de empresas asociadas a la nueva economía. Madrid: Pirámide.

Ley 2/1995, de 23 de marzo, de Sociedades de Responsabilidad Limitada.

Ley 29/1987, de 18 de diciembre, del Impuesto sobre Sucesiones y Donaciones. 
Makeham, W.M. (1873). "On the Integral of Gompertz's function for expressing the values of sums depending upon the contingency of life", J.I.A., 17, pp. 305327.

Mascareñas, J. (2005). Fusiones y adquisiciones. Madrid: McGraw-Hill.

Miller, M. y Modigliani, F. (1961). "Dividend policy, growth, and the valuation of shares", Journal of Business, 34, pp. 411-433.

Modigliani, F. y Merton, M. (1958). "The cost of capital, corporation finance, and the theory of investment", American Economic Review, 48, pp. 261-297.

Morán, R. (2002). Historia del derecho privado, penal y procesal. UNED-Universitas, 2002, vol. 1.

Poterba, J. (1986). "The market valuation of cash dividends: The citizens utilities case reconsidered", Journal of Financial Economics, 15, pp. 395-406.

Sougiannis, T. y Penman, S. (1996). "A comparison of dividend, cash flow and earnings approaches to equity valuation”, Enero 1995, Revisión de abril 1996.

Suárez, A. (2003). Decisiones óptimas de inversión y financiación. Madrid: Pirámide.

Texto Refundido de la Ley de Sociedades Anónimas, aprobado por Real Decreto Legislativo 1564/1989, de 22 de diciembre. 